\title{
EFSUMB Best Published Paper Prize 2021
}

Awarded to Sophie Morse for the publication entitled Rapid Short-pulse Ultrasound Delivers Drugs Uniformly across the Murine Blood-Brain Barrier with Negligible Disruption Journal: Radiology May 2019 291(2): 459-466 